\title{
Experimental data and computational modeling link auxin gradient and development in the Arabidopsis root
}

\author{
Natalie M. Clark ${ }^{\dagger}$, Maria A. de Luis Balaguer ${ }^{\dagger}$ and Rosangela Sozzani*
}

Department of Plant and Microbial Biology, North Carolina State University, Raleigh, NC, USA

\section{Edited by:}

Lucia Colombo, University of Milan, Italy

\section{Reviewed by:}

Jan Petrášek, Institute of

Experimental Botany-Academy of

Sciences of the Czech Republic,

Czech Republic

Idan Efroni, New York University, USA

\section{*Correspondence:}

Rosangela Sozzani, Department of Plant and Microbial Biology, North

Carolina State University, 2577

Thomas Hall, P. O. Box 7612, Raleigh,

NC 27695, USA

e-mail: rsozzan@ncsu.edu

${ }^{\dagger}$ Natalie M. Clark and Maria A. de Luis Balaguer have contributed equally to this work.
The presence of an auxin gradient in the Arabidopsis root is crucial for proper root development and importantly, for stem cell niche (SCN) maintenance. Subsequently, developmental pathways in the root SCN regulate the formation of the auxin gradient. Combinations of experimental data and computational modeling enable the identification of pathways involved in establishing and maintaining the auxin gradient. We describe how the predictive power of these computational models is used to find how genes and their interactions tightly control the formation of an auxin maximum in the SCN. In addition, we highlight known connections between signaling pathways involving auxin and controlling patterning and development in Arabidopsis.

Keywords: Arabidopsis, auxin gradient, root development, stem cell niche, computational modeling

\section{INTRODUCTION}

Hormones are a crucial component of plant developmental pathways, affecting processes such as embryogenesis, growth, organ size, and stress response (Wolters and Jurgens, 2009). Among these hormones is auxin (most frequently found as indole-3-acetic acid, IAA), which contributes to the development, organization, and patterning of the entire plant (Vanneste and Friml, 2009). Specifically, auxin controls the organization and patterning of the root, including the stem cell niche (SCN; Overvoorde et al., 2010). In Arabidopsis, most auxin biosynthesis occurs in tryptophan-dependent pathways, although other pathways have been discovered (for more details about auxin biosynthesis, refer to Tivendale etal., 2014). Once synthesized in the shoot, auxin travels through the root via a number of polar auxin transporters (Bennett etal., 1996; Swarup et al., 2001; Petrasek et al., 2006; Wisniewska et al., 2006). This polar auxin transport results in a gradient of auxin from the shoot with a maximum in the root SCN. This gradient and maximum have been shown to affect root development and patterning (Berleth etal., 2007; Grieneisen et al., 2007; Laskowski et al., 2008). In this review, we first examine how auxin transport proteins and local auxin biosynthesis in particular areas of the root contribute to the gradient and maximum formation. We then address pathways downstream of auxin that affect root patterning, specification of the SCN, and lateral root development. Mathematical modeling is used to predict and eventually validate the proposed models along with experimental data. Finally, we will also discuss these mathematical models to better understand the link between auxin and development.

\section{AUXIN GRADIENT AND MAXIMUM FORMATION IN THE ROOT SCN}

In Arabidopsis, some auxin is synthesized in the shoot (Cheng et al., 2006). Once synthesized, auxin travels from the shoot down to the root tip, where it forms a maximum in the SCN (Grieneisen et al., 2007; Figure 1A). The SCN consists of the quiescent center (QC) surrounded by four types of stem cells. The QC cells are relatively mitotically inactive and produce cellular signals that regulate the maintenance of the stem cells around them (Dolan et al., 1993; Sozzani and Iyer-Pascuzzi, 2014). Auxin transport throughout the root is facilitated by influx and efflux carriers. The AUXIN1/LIKEAUX1 (AUX1/LAX) transporters are the major influx carriers (Bennett etal., 1996; Swarup et al., 2001) while PIN FORMED (PIN) protein family members are the major auxin efflux carriers (Petrasek et al., 2006; Wisniewska et al., 2006; Kleine-Vehn and Friml, 2008). In Arabidopsis, there are eight known PIN proteins: PIN1-PIN8 (Blilou et al., 2005; Mravec et al., 2009; Ding et al., 2012). PIN5, PIN6, and PIN8 belong to a less characterized subclade of PIN proteins and are localized in the endoplasmic reticulum membrane (Mravec et al., 2009). PIN1 is localized mainly at the basal end of vasculature cells. PIN2 is located apically (the end of the cell closest to the shoot) in epidermal and lateral root cap cells and basally in cortical cells. PIN3 is expressed in the columella, at the basal side of vasculature cells, and at the lateral side of the pericycle cells of the elongation zone. PIN4 is localized in the SCN and basally in provascular cells. Finally, PIN7 resides at lateral and basal membranes of provascular cells in the meristem and elongation zone, whereas in the columella cells it coincides with the PIN3 domain. (Figure 1B; Blilou et al., 2005). These various PIN proteins produce diverse auxin flux patterns. Since they 


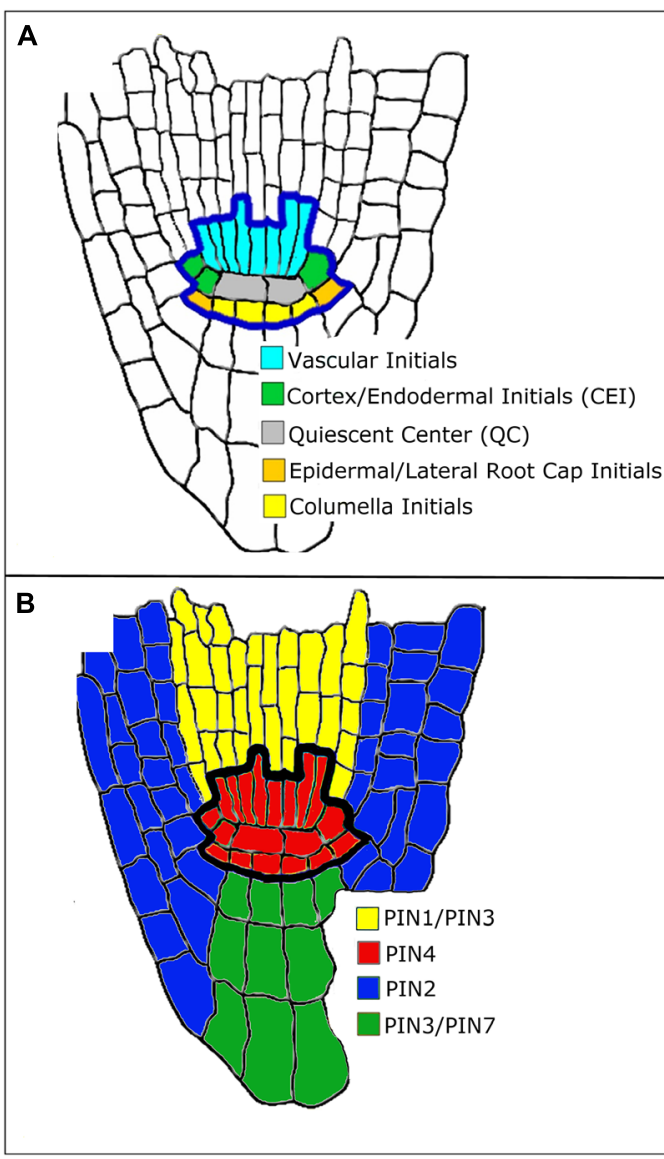

FIGURE 1 | Mechanisms of auxin transport in the Arabidopsis root (A) Root stem cell niche (SCN) organization. (B) PIN protein localization around the SCN. Note that the colors do not represent the exact localization

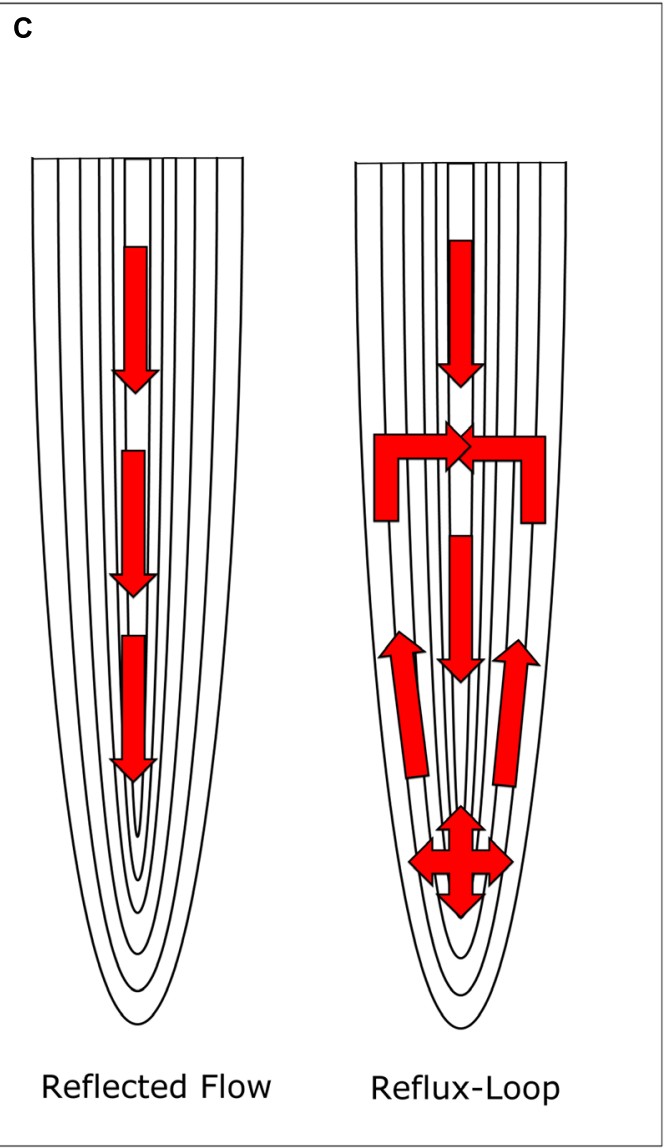

of the PIN protein at the membrane, rather the cell populations where the PIN proteins are located. (C) Reflected flow (Mironova et al., 2010) and reflux-loop (Grieneisen etal., 2007) mechanisms of auxin transport. are located in different cells, this results in different auxin transport rates throughout the root (Blilou et al., 2005). Thus, PIN localization is crucial for the formation of the auxin gradient and maximum (Grieneisen et al., 2007).

Two mechanisms have been proposed to explain how PINs establish and maintain the auxin gradient: the reflux-loop mechanism (Grieneisen et al., 2007) and the reflected flow mechanism (Mironova et al., 2010; Figure 1C). The reflected flow mechanism only considers PIN1 protein localization and, instead of considering each individual cell population, generalizes to vasculature and non-vasculature cells. PIN1 transports auxin down the shoot into the SCN. Once the auxin maximum passes a certain threshold, PIN1 proteins degrade, preventing the transport of auxin down the root and forming the auxin gradient. The reflected flow model is based on the activator-inhibitor mechanism. In this mechanism, pattern formation is induced by both a positive and negative regulator. In the reflected flow model, auxin serves as both the activator and inhibitor of PIN1 protein expression (Mironova et al., 2010). Grieneisen et al. (2007) built upon the reflected flow mechanism by adding PIN2 and PIN3 to the model. In their reflux-loop mechanism, auxin is transported from the vasculature to the root tip. Once the auxin reaches the SCN, PINs transport auxin laterally and then up the root, back to the vasculature. Unlike other models, the reflux-loop mechanism accounts for the spatial structure of cells. This allows diffusion and cell permeability to be described as two separate parameters instead of one combined flux parameter. Additionally this model accounts for specific sizes and shapes of individual cells (Grieneisen et al., 2007). In order to show that their proposed mechanisms produce the auxin gradient and maximum, both of these groups used mathematical modeling. The reflected flow model is a set of ordinary differential equations (ODEs) that measure the change in auxin and PIN protein concentrations over time (Mironova et al., 2010). The reflux-loop model is a set of partial differential equations that measure the change in auxin concentrations over time and space (between the cells of the root). Unlike in the reflected-flow model, the reflux-loop model assumes that PIN transport of auxin is constant (Grieneisen et al., 2012). Both groups then produced computer simulations of their models that illustrate how the equations produce both the auxin gradient and maximum in the SCN (Mironova et al., 2010; Grieneisen et al., 2012). Since both of these methods are able to generate the auxin gradient and maximum, one might suggest that they cooperate together to regulate root development. Indeed, a model that combines both of these mechanisms shows how they regulate root 
apical meristem (RAM) development. Regeneration of the RAM is provided by the reflected flow mechanism while the reverse fountain enables RAM maintenance (Mironova et al., 2012). Crucially, these models depend on a particular PIN localization: the PIN proteins must be located in a specific cell and in a specific orientation. However, the exact distribution of PIN proteins within the root is uncertain since the concentrations and distribution of PIN proteins within the cell can change over time. Auxin is known to regulate PIN distribution, but it is unclear whether auxin concentration, flux, or both contribute to PIN localization (for more on PIN distribution, see Goh et al., 2014). Recently, Tian et al. (2014) adapted the reflux-loop model (Grieneisen et al., 2007 to account for experimentally observed PIN localization (Kleine-Vehn and Friml, 2008). This adapted model cannot produce the auxin maximum in the QC, suggesting that there are other pathways besides PIN-mediated auxin transport that maintain the auxin maximum (Tian et al., 2014).

Another contributing factor to the auxin maximum is the synthesis of auxin in the QC within the SCN of the root (Brady et al., 2007; Stepanova et al., 2008; Petersson et al., 2009). Auxin influx within the root tip is regulated by AUX1, which has been shown to control which tissues have high auxin levels (Band et al., 2014). The addition of local auxin synthesis and AUX1 expression in the root tip improves the previous PIN transport models. In this mathematical model, the auxin maximum in the QC depends on AUX1 expression in the lateral root cap and not on the specific PIN localization in the epidermis (Band et al., 2014). Moreover, auxin production and transport within the root tip is a distinct process from auxin production and transport from the shoot. Overproduction of auxin in the shoot cannot rescue auxin deficiency in the root tip (Chen et al., 2014). Thus, transport of auxin through AUX1 as well as local synthesis of auxin in the root tip contributes to the formation of the auxin gradient and maximum.

One important component of these mathematical models is the measurement of auxin levels within the Arabidopsis root. Both the DR5 promoter and the DII-VENUS sensor can be used as proxies for auxin content. The DR5 promoter drives expression of a reporter gene, such as $\beta$-glucuronidase (GUS) that allows one to measure auxin production and distribution (Ulmasov et al., 1997; Sabatini et al., 1999). The auxin-interaction domain DII drives the fast maturing yellow fluorescent protein VENUS. Unlike DR5, DII-VENUS rapidly degrades in the presence of auxin (Brunoud et al., 2012). Importantly, these two auxin reporters have different expression patterns: while $D R 5$ expression fits the traditional auxin gradient with a maximum in the QC, DII-VENUS expression suggests that there are cell type-specific differences in auxin distribution (Ulmasov et al., 1997; Sabatini et al., 1999; Brunoud et al., 2012). Recently, an auxin-responsive transcriptomic analysis found that auxin response genes fit one of two categories: either the genes are characterized by high expression in the root tip with peaks toward the shoot, or the genes have a graded meristematic response. While the first of these groups fits the stereotypical auxin gradient, the second suggests that there could be other cell type-specific auxin gradients (Bargmann et al., 2013). In the future, it would be interesting to study how the addition of multiple auxin gradients affects the previously developed mathematical models.
It is clear that the formation of the auxin gradient and maximum does not depend on one pathway but on a number of pathways. Auxin is produced both in the shoot and in the SCN. The PIN proteins are responsible for transporting auxin from the shoot down to the SCN, while AUX1 transports auxin throughout the SCN. The key to determining that all of these mechanisms are necessary is the use of mathematical and computational modeling. When the mathematical models for the PIN transport pathway were unable to generate the auxin maximum under all conditions, new models were developed to tie in AUX1-mediated transport and auxin production in the root tip. The next logical step is to use this same mathematical modeling approach to address auxin's role in root development.

\section{AUXIN ROLE IN ACD MECHANISMS AND ROOT DEVELOPMENT}

The pathways in which auxin has been reported to be involved occur in a wide range of developmental processes, including the embryonic and postembryonic SCN specification and maintenance, and the initiation and growth of new organs as lateral root initiation and development (Casimiro et al., 2001; Marchant et al., 2002; Laskowski et al., 2008; Swarup et al., 2008; Yoshida et al., 2014). Auxin gradient, decreasing from the QC toward the transition zone ( $\mathrm{TZ}$-between the apical meristem and basal elongation region), plays a role in controlling meristem patterning and maintenance. In particular, auxin acts with another hormone, cytokinin, to ensure a balance between cell division, promoted by auxin (Blilou et al., 2005) and cell differentiation, promoted by cytokinin (Ioio et al., 2008). Through the upregulation of the expression of tyrosylprotein sulfotransferases (TPSTs), auxin also affects growth factors in Arabidopsis, such as phytosulfokine peptides and root meristem growth factors (RGFs; Zhou et al., 2010). The auxin maximum and gradient contribute to positioning asymmetric cell divisions (ACDs) in a specific tissue layer in the root (Cruz-Ramírez et al., 2012) and are related to SCN specification (Sabatini et al., 1999, 2003; Benjamins and Scheres, 2008; Dinneny and Benfey, 2008; Zhou etal., 2010). Mechanisms such as those involved in ACDs positioning and meristem maintenance often have reciprocal influences on the formation of auxin maximum and gradient (Cruz-Ramírez et al., 2012; Moubayidin et al., 2013; Tian et al., 2014). Computational modeling is a powerful tool, necessary to test complex biological hypotheses, that allows an understanding of the interactions among the elements involved in the pathways (Grieneisen and Scheres, 2009). It also allows us to merge knowledge gained from different mechanisms, facilitating our understanding of the connections between multiple pathways involved in development (Narula et al., 2013). In this section, we review some root development mechanisms involving auxin, with a special focus on those influencing the SCN. We highlight new root development mechanisms involving auxin where mathematical and/or computational modeling has been integrated (Laskowski et al., 2008; Jones et al., 2009; Cruz-Ramírez etal., 2012; Tian etal., 2014).

The role of auxin in root stem cell specification in zygotic embryogenesis has long been known (Friml et al., 2003; Weijers et al., 2005; Breuninger et al., 2008). Local auxin synthesis in the 
SCN regulates PIN1 polarization and the auxin maximum, resulting in proper cell polarization during embryogenesis (Robert et al., 2013; Wabnik et al., 2013). Auxin activates feedback repressors of cytokinin signaling, the ARABIDOPSIS RESPONSE REGULATOR genes ARR7 and ARR15 (Müller and Sheen, 2008). Loss of function of these genes in the basal cell during early embryogenesis results in a defective root stem cells, indicating that the interaction between auxin and cytokinin is critical for specifying root stem cell niche (Müller and Sheen, 2008). It was more recently shown that auxin influx carriers play a role in patterning of the embryonic root (Ugartechea-Chirino et al., 2010). The results in this study indicated redundancy within the AUX1 LAX family in the establishment of cell organization in the radicle apex of arabidopsis.

During post-embryonic development, auxin is also related to SCN positioning, patterning and specification (Sabatini et al., 1999, 2003; Zhou et al., 2010). Specifically, auxin stimulates the transcription of genes PLETHORA1 (PLT1) and PLT2, which encode members of the AP2 class of transcription factors (Aida et al., 2004). These genes are critical for stem cell specification and maintenance, from the basal embryo to the embryonic root up to post-embryonic root meristem (Aida et al., 2004). PLTs are necessary for root formation and for mediating the developmental response to auxin in the root (Galinha et al., 2007). PLTs act in an interaction network with PIN proteins to control patterning and growth of the root and to stabilize the position of the SCN (Blilou et al., 2005; Dinneny and Benfey, 2008). Auxin upregulates expression of the Arabidopsis TPST gene (Zhou et al., 2010), which affects expression of PLT1, PLT2, PIN genes, and auxin biosynthetic genes, and is believed to maintain root SCN through the root meristem growth factor RGF1 (Matsuzaki et al., 2010). Loss of TPST function leads to defects in the root SCN and root growth and development (Komori et al., 2009; Zhou et al., 2010), thus linking auxin, PINs, PLTs, and TPST acting in a postembryonic SCN maintenance mechanism.

Previous experimental observations had concluded that PIN proteins are localized at the apical side of epidermis cells and to the basal-outer lateral side of cortex cells (Kleine-Vehn and Friml, 2008; Wabnik et al., 2011). Tian et al. (2014) incorporated these observations into a simplified version of the reflux-loop root computer model (Grieneisen et al., 2007), which resulted in a model that could not reproduce the auxin accumulation in the QC cells as observed experimentally (Tian et al., 2014). This suggested additional mechanisms in auxin maxima localization. In addition to auxin, the transcription factor WUSCHEL-related homeobox 5 (WOX5) is also believed to participate in SCN maintenance (Sarkar et al., 2007). Subsequent experimental observations and computational simulations by Tian et al. (2014) indicated that WOX5 modulates expression of auxin biosynthetic genes in the QC. It was also found that this is balanced through the activity of IAA17 auxin response repressor (Tian et al., 2014). A loop that connects auxin and WOX5, the WOX5-IAA17 feedback circuit, was thus proposed. The WOX5-IAA17 loop was shown to contribute to the maintenance of auxin maximal responses in the QC cells, and, in turn, to affect the patterning of the root SCN (Tian et al., 2014). Specifically, the predictive model developed by Tian et al. (2014) aimed to describe the contribution of the WOX5-IAA17 feedback circuit to the maintenance of auxin maximal responses in the QC. The model consists of a cellular grid tissue template, generated with the VV (Vertex-Vertex) programming language (Smith et al., 2006), and a set of coupled ODEs. The model predicted (1) a WOX5 expression maximum in the QC cells, which contributed to auxin production and maintenance of auxin maximum in these cells and (2) the decrease of auxin concentration from the QC toward distal columella cells. These computational simulations matched the experimental results, which indicated that the WOX5-IAA17 circuit might be responsible for auxin maximum maintenance in the root tip and in turn for root stem cell differentiation. The predictions made by the model thus, were observed experimentally.

This auxin maximum in the QC was also shown to regulate localization of the ACDs along with the SHORTROOTSCARECROW (SHR-SCR) pathway (Cruz-Ramírez et al., 2012; see Figure 2). Specifically, the RETINOBLASTOMA-RELATED (RBR) protein binds the stem cell regulator SCR. RBR is phosphorylated through a CYCLIND6;1-CDK complex, which hinders SCR activity. CYCD6; 1 is at the same time transcriptionally regulated by a complex containing SCR and SHR, which creates a positive feedback loop. Auxin maximum positively regulates transcription of CYCD6;1 as well, constraining the ACDs to the SCN. Cruz-Ramírez et al. (2012) developed a predictive model to unravel this mechanism, revealing the existence of a double positive feedback loop that grants this system with a switch-like ACD behavior. The model describes a nested feedback circuit representing the spatiotemporal control of ACDs, integrating auxin-mediated tissue polarity in the longitudinal developmental axis (Grieneisen et al., 2007) with the SHR expression pattern in the radial developmental axis. The model consists of a set of coupled ODEs that capture the dynamics of the key components within each cell, which are in turn integrated within the tissue context. Through a combination of this model and experimental

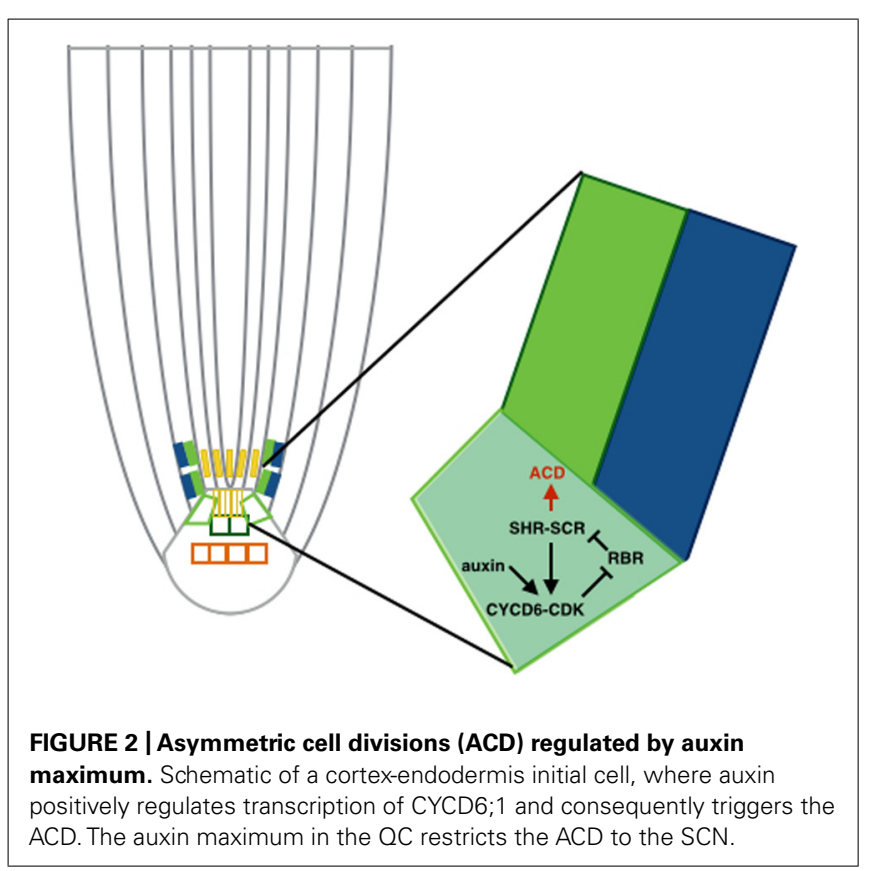


data, the authors show that auxin and SHR influx trigger the ACD state of the bistable circuit and the radial confinement of SHR. All the predictions made by the model are consistent with the data from this study.

In addition to regulating localization of the ACD mechanism within the SHR-SCR pathway, SCR has been shown to be responsible for other root developmental aspects. SCR in the QC cells controls auxin production, thus governing cell division of the surrounding stem cells and, at the same time, the rate of cell differentiation of meristematic daughter cells at the TZ (Moubayidin et al., 2013). Moubayidin et al. (2013) proposed a descriptive model that, based on their observations, reflected the dependence of root meristem activity on SCR, although no mathematical description was provided. The model particularly shows how SCR directly suppresses the cytokinin-response transcription factor ARR1 in the QC cells, thus titrating local auxin production and stem cell division. Auxin produced in the QC, via polar auxin transport machinery, activates ARR1 at the transition zone, thus committing meristematic cells to differentiate. This model proposes a single pivotal gene, SCR, which orchestrates a reciprocal cross talk between cytokinin and auxin. This mechanism contributes to form the meristem zonation along the apical-basal axes, coordinating cell division with cell differentiation at the opposite ends of root meristem, thus leading to a correct root organ growth.

Another role for cytokinin and auxin in the SCN specification was recently elucidated (Zhang et al., 2013). Cytokinin adjusts auxin distribution in the RAM via the auxin influx and efflux carriers, PINs and AUX/LAX, consequently regulating the mitotic activity in the QC (Zhang etal., 2013). Specifically, the authors showed that cytokinin affects the auxin response in the QC via downregulation of LAX2, in turn negatively regulating QC specification.

There is also evidence of roles for auxin in root development outside the SCN. For example, auxin has been related to lateral root initiation and development (Xie et al., 2000; Casimiro et al., 2001; Marchant et al., 2002; Swarup et al., 2008; De Smet et al., 2010), and the crosstalk between auxin and cytokinin is involved in radial pattern of the root, vascular differentiation, root gravitropism, and together with ethylene, lateral root initiation (Aloni et al., 2006). NAC1, a member of the NAC family, is induced by auxin and activates expression of two downstream auxin-responsive genes to promote lateral root development (Xie et al., 2000). It was later proposed that auxin enters cortical and epidermal cells underlying new lateral root primordia, inducing the expression of auxin influx carrier LAX3 (Swarup et al., 2008). Increased LAX3 facilitates auxin uptake, reinforcing expression of LAX3. This feedback loop results in auxin accumulation that triggers cell wall remodeling genes (Swarup et al., 2008). Other experiments showed that auxin accumulates on the outside of the vasculature in bent regions of the root (Laskowski et al., 2008). These areas correspond to AUX1 up-regulation, PIN down-regulation, and maxima of lateral root initiation regions. This suggests that auxin influx plays a major role in determining the density of root primordia (Laskowski et al., 2008). In this study, Laskowski et al. (2008) used a predictive, computational model focused on describing the dynamics of auxin transport through the root and understanding the impact of curvatures in auxin accumulation and lateral root formation.
Specifically, the model included cell geometries, typical PIN distributions, changes in cell-shapes caused by curvature of the root, and auxin transport. The model was able to predict that curvature of the root induces auxin accumulation on the outside of the vasculature in bent regions, where lateral root initiation is highly likely to occur. These studies suggested that auxin was responsible for initiation of LR primordia and the spatial patterning of lateral organs in roots. Recent studies, however, suggested that both LR positioning and root bending are periodic responses that may be regulated by an oscillating gene expression mechanism (MorenoRisueno et al., 2010; Van Norman et al., 2014). Moreno-Risueno et al. (2010) indicated that although auxin may be involved in the process, it does not seem to be sufficient to initiate prebranch sites. Instead, their data suggested that oscillating genes establish the temporal and spatial distribution of LRs along the primary root axis. These findings were later restated by Van Norman et al. (2014), where carotenoids were additionally found to be necessary for the output of the LR clock and establishment of the LR prepattern. This suggests that LR formation may be simultaneously regulated at several molecular levels, although a relationship between hormones such as auxin and the endogenous clock-like mechanism underlying LR patterning has not yet been reported.

Another important aspect of root development in which auxin is known to take part is initiation and growth of root hairs (Masucci and Schiefelbein, 1994; Pitts et al., 1998; Rahman et al., 2002; Jones et al., 2009). It has been suggested that insensitivity to ethylene affects the auxin-driven root hair elongation, placing the hormones auxin and ethylene in another crosstalk pathway that plays a role in root hair development (Rahman et al., 2002). It was later revealed that the auxin-influx transporter AUX1 is not present in root-hair cells, but it is instead highly expressed in adjacent non-hair cell files (Jones et al., 2009). Jones et al. (2009) used a three dimensional model of auxin flow in the root's tip, which suggests that transport of auxin from non-hair cells maintains an auxin supply to hair cells during their growth. Together, these results clearly indicate the involvement of auxin and auxin transport in root hair initiation and development.

\section{CONCLUDING REMARKS}

Recent studies have described the predicted roles of auxin in SCN specification, patterning, and maintenance, as well as its contribution to root growth, lateral root initiation, and root hair development. The reciprocal influences of pathways affecting the formation of an auxin maximum and gradient, and this gradient affecting root development, have been highlighted. Elucidating the mechanisms by which signaling pathways involving auxin and controlling patterning and development is a key challenge for understanding the systems biology of plant growth. Due to its polar movement, it is difficult to predict the auxin dynamics by studying it locally. The computational models above presented have yielded advances in our understanding of the role of auxin in development, allowing more global and intuitive interpretations of diverse data and results. Thus, the use of computational models as a tool to study and understand mechanisms underlying root development has enabled such systems-level modeling. Identifying critical features governing such regulatory networks and their integration will be the next necessary step toward a predictive 
mechanistic model. An accurate characterization of the quantitative parameters will therefore be essential to generate models that accurately capture the behavior of the system. This will allow us to identify and to predict decision-making signals, ultimately leading to control of plant growth and development.

\section{ACKNOWLEDGMENTS}

Work in the Sozzani lab is funded by the North Carolina Agricultural Research Service and North Carolina State University Provost's Office. Natalie M. Clark is supported by the Mathematical Biology Research Training Group. We thank Laila Moubayidin, Jaimie Van Norman, Linda Robles, Robert Franks, and Riccardo Di Mambro for critical reading of this manuscript.

\section{REFERENCES}

Aida, M., Beis, D., Heidstra, R., Willemsen, V., Blilou, I., Galinha, C., et al. (2004). The PLETHORA genes mediate patterning of the Arabidopsis root stem cell niche. Cell 119, 109-120. doi: 10.1016/j.cell.2004.09.018

Aloni, R., Aloni, E., Langhans, M., and Ullrich, C. (2006). Role of cytokinin and auxin in shaping root architecture: regulating vascular differentiation, lateral root initiation, root apical dominance and root gravitropism. Ann. Bot. 97, 883-893. doi: $10.1093 / \mathrm{aob} / \mathrm{mcl} 027$

Band, L. R., Wells, D. M., Fozard, J. A., Ghetiu, T., French, A. P., Pound, M. P., et al. (2014). Systems analysis of auxin transport in the Arabidopsis root apex. Plant Cell 26, 862-875. doi: 10.1105/tpc.113.119495

Bargmann, B. O. R., Vanneste, S., Krouk, G., Nawy, T., Efroni, I., Shani, E., et al. (2013). A map of cell type-specific responses. Mol. Syst. Biol. 9, 688. doi: $10.1038 / \mathrm{msb} .2013 .40$

Benjamins, R., and Scheres, B. (2008). Auxin: the looping star in plant development. Annu. Rev. Plant Biol. 59, 443-465. doi: 10.1146/annurev.arplant.58.032806.103805

Bennett, M. J., Marchant, A., Green, H. G., May, S. T., Ward, S. P., Millner, P. A., et al. (1996). Arabidopsis AUX1 gene: a permease-like regulator of root gravitropism. Science 273, 948-950. doi: 10.1126/science.273.5277.948

Berleth, T., Scarpella, E., and Prusinkiewicz, P. (2007). Towards the systems biology of auxin-transport-mediated patterning. Trends Plant Sci. 12, 151-159. doi: 10.1016/j.tplants.2007.03.005

Blilou, I., Xu, J., Wildwater, M., Willemsen, V., Paponov, I., Friml, J., et al. (2005). The PIN auxin efflux facilitator network controls growth and patterning in Arabidopsis roots. Nature 433, 39-44. doi: 10.1038/nature03184

Brady, S. M., Orlando, D. A., Lee, J.-Y., Wang, J. Y., Koch, J., Dinneny, J. R., et al. (2007). A high-resolution root spatiotemporal map reveals dominant expression patterns. Science 318, 801-806. doi: 10.1126/science.1146265

Breuninger, H., Rikirsch, E., Hermann, M., Ueda, M., and Laux, T. (2008). Differential expression of WOX genes mediates apical-basal axis formation in the Arabidopsis embryo. Dev. Cell 14, 867-876. doi: 10.1016/j.devcel.2008.03.008

Brunoud, G., Wells, D. M., Oliva, M., Larrieu, A., Mirabet, V., Burrow, A. H., et al. (2012). A novel sensor to map auxin response and distribution at high spatio-temporal resolution. Nature 482, 103-108. doi: 10.1038/nature 10791

Casimiro, I., Marchant, A., Bhalerao, R. P., Beeckman, T., Dhooge, S., Swarup, R., et al. (2001). Auxin transport promotes Arabidopsis lateral root initiation. Plant Cell 13, 843-852. doi: 10.1105/tpc.13.4.843

Chen, Q., Dai, X., De-Paoli, H., Cheng, Y., Takebayashi, Y., Kasahara, H., et al. (2014). Auxin overproduction in shoots cannot rescue auxin deficiencies in Arabidopsis roots. Plant Cell Physiol. 55, 1072-1079. doi: 10.1093/pcp/pcu039

Cheng, Y., Dai, X., and Zhao, Y. (2006). Auxin biosynthesis by the YUCCA flavin monooxygenases controls the formation of floral organs and vascular tissues in Arabidopsis. Genes Dev. 20, 1790-1799. doi: 10.1101/gad.1415106

Cruz-Ramírez, A., Díaz-Triviño, S., Blilou, I., Grieneisen, V. A., Sozzani, R., Zamioudis, C., et al. (2012). A bistable circuit involving SCARECROWRETINOBLASTOMA integrates cues to inform asymmetric stem cell division. Cell 150, 1002-1015. doi: 10.1016/j.cell.2012.07.017

De Smet, I., Lau, S., Voß, U., Vanneste, S., Benjamins, R., Rademacher, E. H., et al. (2010). Bimodular auxin response controls organogenesis in Arabidopsis. Proc. Natl. Acad. Sci. U.S.A. 107, 2705-2710. doi: 10.1073/pnas.0915001107
Ding, Z., Wang, B., Moreno, I., Duplakova, N., Simon, S., Carraro, N., et al. (2012). ER-localized auxin transporter PIN8 regulates auxin homeostasis and male gametophyte development in Arabidopsis. Nat. Commun. 3, 1-9. doi: 10.1038/ncomms1941

Dinneny, J. R., and Benfey, P. N. (2008). Plant stem cell niches: standing the test of time. Cell 132, 553-557. doi: 10.1016/j.cell.2008.02.001

Dolan, L., Janmaat, K., Wilemsen, V., Linstead, P., Poething, S., Roberts, K., et al. (1993). Cellular organisation of the Arabidopsis thaliana root. Development 119, 71-84.

Friml, J., Vieten, A., Sauer, M., Weijers, D., Schwarz, H., Hamann, T., et al. (2003). Efflux-dependent auxin gradients establish the apical-basal axis of Arabidopsis. Nature 426, 147-153. doi: 10.1038/nature02085

Galinha, C., Hofhuis, H., Luijten, M., Willemsen, V., Blilou, I., Heidstra, R., et al. (2007). PLETHORA proteins as dose-dependent master regulators of Arabidopsis root development. Nature 449, 1053-1057. doi: 10.1038/nature06206

Goh, T., Voß, U., Farcot, E., Bennett, M. J., and Bishop, A. (2014). Systems biology approaches to understand the role of auxin in root growth and development. Physiol. Plant. 151, 73-82. doi: 10.1111/ppl.12162

Grieneisen, V. A., and Scheres, B. (2009). Back to the future: evolution of computational models in plant morphogenesis. Curr. Opin. Plant Biol. 12, 606-614. doi: 10.1016/j.pbi.2009.07.008

Grieneisen, V. A., Scheres, B., Hogeweg, P., and Maree, A. F. M. (2012). Morphogengineering roots: comparing mechanisms of morphogen gradient formation. BMC Syst. Biol. 6:37. doi: 10.1186/1752-0509-6-37

Grieneisen, V. A., Xu, J., Marée, A. F., Hogeweg, P., and Scheres, B. (2007). Auxin transport is sufficient to generate a maximum and gradient guiding root growth. Nature 449, 1008-1013. doi: 10.1038/nature06215

Ioio, R. D., Nakamura, K., Moubayidin, L., Perilli, S., Taniguchi, M., Morita, M. T., etal. (2008). A genetic framework for the control of cell division and differentiation in the root meristem. Science 322, 1380-1384. doi: $10.1126 /$ science. 1164147

Jones, A. R., Kramer, E. M., Knox, K., Swarup, R., Bennett, M. J., Lazarus, C. M., et al. (2009). Auxin transport through non-hair cells sustains root-hair development. Nat. Cell Biol. 11, 78-84. doi: 10.1038/ncb1815

Kleine-Vehn, J., and Friml, J. (2008). Polar targeting and endocytic recycling in auxin-dependent plant development. Annu. Rev. Cell Dev. Biol. 24, 447-473. doi: 10.1146/annurev.cellbio.24.110707.175254

Komori, R., Amano, Y., Ogawa-Ohnishi, M., and Matsubayashi, Y. (2009). Identification of tyrosylprotein sulfotransferase in Arabidopsis. Proc. Natl. Acad. Sci. U.S.A. 106, 15067-15072. doi: 10.1073/pnas.0902801106

Laskowski, M., Grieneisen, V. A., Hofhuis, H., Colette, A., Hogeweg, P., Marée, A. F., et al. (2008). Root system architecture from coupling cell shape to auxin transport. PLoS Biol. 6:e307. doi: 10.1371/journal.pbio.0060307

Marchant, A., Bhalerao, R., Casimiro, I., Eklöf, J., Casero, P. J., Bennett, M., et al. (2002). AUX1 promotes lateral root formation by facilitating indole-3-acetic acid distribution between sink and source tissues in the Arabidopsis seedling. Plant Cell 14, 589-597. doi: 10.1105/tpc.010354

Masucci, J. D., and Schiefelbein, J. W. (1994). The rhd6 mutation of Arabidopsis thaliana alters root-hair initiation through an auxin-and ethylene-associated process. Plant Physiol. 106, 1335-1346.

Matsuzaki, Y., Ogawa-Ohnishi, M., Mori, A., and Matsubayashi, Y. (2010). Secreted peptide signals required for maintenance of root stem cell niche in Arabidopsis. Science 329, 1065-1067. doi: 10.1126/science.1191132

Mironova, V. V., Omelyanchuk, N. A., Novoselova, E. S., Doroshkov, A. V., Kazantsev, F. V., Kochetov, A. V., et al. (2012). Combined in silico/in vivo analysis of mechanism providing for root apical meristem self-organization and maintenance. Ann. Bot. 110, 349-360. doi: 10.1093/aob/mcs069

Mironova, V. V., Omelyanchuk, N. A., Yosiphon, G., Fadeev, S. I., Kolchanov, N. A., Mjolsness, E., et al. (2010). A plausible mechanism for auxin patterning along the developing root. BMC Syst. Biol. 4:98. doi: 10.1186/1752-0509-4-98

Moreno-Risueno, M. A., Van Norman, J. M., Moreno, A., Zhang, J., Ahnert, S. E., and Benfey, P. N. (2010). Oscillating gene expression determines competence for periodic Arabidopsis root branching. Science 329, 1306-1311. doi: 10.1126/science.1191937

Moubayidin, L., Di Mambro, R., Sozzani, R., Pacifici, E., Salvi, E., Terpstra, I., et al. (2013). Spatial coordination between stem cell activity and cell differentiation in the root meristem. Dev. Cell 26, 405-415. doi: 10.1016/j.devcel.2013. 06.025 
Mravec, J., Skupa, P., Bailly, A., Hoyerova, K., Krecek, P., Bielach, A., et al. (2009). Subcellular homeostasis of phytohormone auxin is mediated by the ER-localized PIN5 transporter. Nature 459, 1136-1140. doi: 10.1038/nature08066

Müller, B., and Sheen, J. (2008). Cytokinin and auxin interaction in root stemcell specification during early embryogenesis. Nature 453, 1094-1097. doi: 10.1038 /nature 06943

Narula, J., Williams, C., Tiwari, A., Marks-Bluth, J., Pimanda, J. E., and Igoshin, O. A. (2013). Mathematical model of a gene regulatory network reconciles effects of genetic perturbations on hematopoietic stem cell emergence. Dev. Biol. 379, 258-269. doi: 10.1016/j.ydbio.2013.04.016

Overvoorde, P., Fukaki, H., and Beeckman, T. (2010). Auxin control of root development. Cold Spring Harb. Perspect. Biol. 2, a001537. doi: 10.1101/cshperspect.a001537

Petersson, S. V., Johansson, A. I., Kowalczyk, M., Makoveychuk, A., Wang, J. Y., Moritz, T., et al. (2009). An auxin gradient and maximum in the Arabidopsi root apex shown by high-resolution cell-specific analysis of IAA distribution and synthesis. Plant Cell 21, 1659-1668. doi: 10.1105/tpc.109.066480

Petrasek, J., Mravec, J., Bouchard, R., Blakeslee, J. J., Abas, M., Seifertova, D., et al. (2006). PIN proteins perform a rate-limiting function in cellular auxin efflux. Science 312, 914-918. doi: 10.1126/science.1123542

Pitts, R. J., Cernac, A., and Estelle, M. (1998). Auxin and ethylene promote root hair elongation in Arabidopsis. Plant J. 16, 553-560. doi: 10.1046/j.1365313x.1998.00321.x

Rahman, A., Hosokawa, S., Oono, Y., Amakawa, T., Goto, N., and Tsurumi, S. (2002). Auxin and ethylene response interactions during Arabidopsis root hair development dissected by auxin influx modulators. Plant Physiol. 130, 1908-1917. doi: 10.1104/pp.010546

Robert, H. S., Grones, P., Stepanova, A. N., Robles, L. M., Lokerse, A. S., Alonso, J M., et al. (2013). Local auxin sources orient the apical-basal axis in Arabidopsis embryos. Curr. Biol. 23, 2506-2512. doi: 10.1016/j.cub.2013.09.039

Sabatini, S., Beis, D., Wolkenfelt, H., Murfett, J., Guilfoyle, T., Malamy, J., et al. (1999). An auxin-dependent distal organizer of pattern and polarity in the Arabidopsis root. Cell 99, 463-472. doi: 10.1016/S0092-8674(00)81535-4

Sabatini, S., Heidstra, R., Wildwater, M., and Scheres, B. (2003). SCARECROW is involved in positioning the stem cell niche in the Arabidopsis root meristem. Genes Dev. 17, 354-358. doi: 10.1101/gad.252503

Sarkar, A. K., Luijten, M., Miyashima, S., Lenhard, M., Hashimoto, T., Nakajima, K. et al. (2007). Conserved factors regulate signalling in Arabidopsis thaliana shoot and root stem cell organizers. Nature 446, 811-814. doi: 10.1038/nature05703

Smith, R. S., Guyomarc'h, S., Mandel, T., Reinhardt, D., Kuhlemeier, C., and Prusinkiewicz, P. (2006). A plausible model of phyllotaxis. Proc. Natl. Acad. Sci. U.S.A. 103, 1301-1306. doi: 10.1073/pnas.0510457103

Sozzani, R., and Iyer-Pascuzzi, A. (2014). Postembryonic control of roo meristem growth and development. Curr. Opin. Plant Biol. 17, 7-12. doi: 10.1016/j.pbi.2013.10.005

Stepanova, A. N., Robertson-Hoyt, J., Yun, J., Benavente, L. M., Xie, D.-Y., Dolezal, K., etal. (2008). TAA1-mediated auxin biosynthesis is essential for hormone crosstalk and plant development. Cell 133, 177-191. doi: 10.1016/j.cell.2008.01.047

Swarup, K., Benková, E., Swarup, R., Casimiro, I., Péret, B., Yang, Y., et al. (2008). The auxin influx carrier LAX3 promotes lateral root emergence. Nat. Cell Biol. 10, 946-954. doi: 10.1038/ncb1754

Swarup, R., Friml, J., Marchant, A., Ljung, K., Sandberg, G., Palme, K., et al. (2001). Localization of the auxin permease AUX1 suggests two functionally distinct hormone transport pathways operate in the Arabidopsis root apex. Genes Dev. 15, 2648-2653. doi: 10.1101/gad.210501

Tian, H., Wabnik, K., Niu, T., Li, H., Yu, Q., Pollmann, S., et al. (2014) WOX5-IAA17 feedback circuit-mediated cellular auxin response is crucial for the patterning of root stem cell niches in Arabidopsis. Mol. Plant 7, 277-289. doi: $10.1093 / \mathrm{mp} / \mathrm{sst} 118$

Tivendale, N. D., Ross, J. J., and Cohen, J. D. (2014). The shifting paradigms of auxin biosynthesis. Trends Plant Sci. 19, 44-51. doi: 10.1016/j.tplants.2013.09.012
Ugartechea-Chirino, Y., Swarup, R., Swarup, K., Péret, B., Whitworth, M., Bennett, M., et al. (2010). The AUX1 LAX family of auxin influx carriers is required for the establishment of embryonic root cell organization in Arabidopsis thaliana. Ann. Bot. 105, 277-289. doi: 10.1093/aob/mcp287

Ulmasov, T., Murfett, J., Hagen, G., and Guilfoyle, T. J. (1997). Aux/lAA proteins repress expression of reporter gene containing natural, and highly active synthetic auxin response elements. Plant Cell 9, 1963-1971. doi: 10.1105/tpc.9.11. 1963

Van Norman, J. M., Zhang, J., Cazzonelli, C. I., Pogson, B. J., Harrison, P. J., Bugg, T. D., et al. (2014). Periodic root branching in Arabidopsis requires synthesis of an uncharacterized carotenoid derivative. Proc. Natl. Acad. Sci. U.S.A. 111, E1300-E1309. doi: 10.1073/pnas.1403016111

Vanneste, S., and Friml, J. (2009). Auxin: a trigger for change in plant development. Cell 136, 1005-1016. doi: 10.1016/j.cell.2009.03.001

Wabnik, K., Govaerts, W., Friml, J., and Kleine-Vehn, J. (2011). Feedback models for polarized auxin transport: an emerging trend. Mol. Biosyst. 7, 2352-2359. doi: 10.1039/c1mb05109a

Wabnik, K., Robert, H. S., Smith, R. S., and Friml, J. (2013). Modeling framework for the establishment of the apical-basal embryonic axis in plants. Curr. Biol. 23, 2513-2518. doi: 10.1016/j.cub.2013.10.038

Weijers, D., Sauer, M., Meurette, O., Friml, J., Ljung, K., Sandberg, G., et al. (2005). Maintenance of embryonic auxin distribution for apical-basal patterning by PINFORMED-dependent auxin transport in Arabidopsis. Plant Cell 17, 2517-2526. doi: 10.1105/tpc.105.034637

Wisniewska, J., Xu, J., Seifertova, D., Brewer, P. B., Ruzicka, K., Blilou, I., et al. (2006). Polar PIN localization directs auxin flow in plants. Science 312, 883. doi: $10.1126 /$ science. 1121356

Wolters, H., and Jurgens, G. (2009). Survival of the flexible: hormonal growth control and adaptation in plant development. Nat. Rev. Genet. 10, 305-317. doi: $10.1038 / \mathrm{nrg} 2558$

Xie, Q., Frugis, G., Colgan, D., and Chua, N.-H. (2000). Arabidopsis NAC1 transduces auxin signal downstream of TIR1 to promote lateral root development. Genes Dev. 14, 3024-3036. doi: 10.1101/gad.852200

Yoshida, S., Barbier De Reuille, P., Lane, B., Bassel, G. W., Prusinkiewicz, P., Smith, R. S., et al. (2014). Genetic control of plant development by overriding a geometric division rule. Dev. Cell 29, 75-87. doi: 10.1016/j.devcel.2014.02.002

Zhang, W., Swarup, R., Bennett, M., Schaller, G. E., and Kieber, J. J. (2013). Cytokinin induces cell division in the quiescent center of the Arabidopsis root apical meristem. Curr. Biol. 23, 1979-1989. doi: 10.1016/j.cub.2013. 08.008

Zhou, W., Wei, L., Xu, J., Zhai, Q., Jiang, H., Chen, R., et al. (2010). Arabidopsis tyrosylprotein sulfotransferase acts in the auxin/PLETHORA pathway in regulating postembryonic maintenance of the root stem cell niche. Plant Cell 22, 3692-3709. doi: $10.1105 /$ tpc. 110.075721

Conflict of Interest Statement: The authors declare that the research was conducted in the absence of any commercial or financial relationships that could be construed as a potential conflict of interest.

Received: 02 May 2014; paper pending published: 29 May 2014; accepted: 23 June 2014; published online: 07 July 2014.

Citation: Clark NM, de Luis Balaguer MA and Sozzani R (2014) Experimental data and computational modeling link auxin gradient and development in the Arabidopsis root. Front. Plant Sci. 5:328. doi: 10.3389/fpls.2014.00328

This article was submitted to Plant Evolution and Development, a section of the journal Frontiers in Plant Science.

Copyright (c) 2014 Clark, de Luis Balaguer and Sozzani. This is an open-access article distributed under the terms of the Creative Commons Attribution License (CC BY). The use, distribution or reproduction in other forums is permitted, provided the original author(s) or licensor are credited and that the original publication in this journal is cited, in accordance with accepted academic practice. No use, distribution or reproduction is permitted which does not comply with these terms. 